\section{Gossypium barbadense: revaloración y sustentabilidad}

Héctor Lozano Gonzales ${ }^{(1)}$, Julia Pelaez Cavero $^{(2)}$ y Laura Bernabé Soto ${ }^{(3)}$

Resumen: El ensayo aborda la problemática del algodón nativo de color peruano y su uso en la elaboración de productos artesanales que no logran competir con el algodón comercial. Pese a ello las familias de las comunidades productoras, ven en el algodón una extensión cultural a mantener vigente, siendo esto insuficiente para su valorización. El objetivo es proponer la revaloración del algodón, a partir de la comunicación de sus virtudes genéticas, el beneficio ambiental en su producción y la innovación en diseño hacia el mercado contemporáneo, procurando que su esencia precolombina potencie el desarrollo económico de las comunidades productoras dentro del marco de la sustentabilidad e identidad.

Palabras claves: algodón nativo - comunidades productoras - artesanía - diseño - sustentabilidad - revalorización.

[Resúmenes en inglés y portugués en las páginas 143-144]

(1) Héctor Lozano Gonzáles, maestro en educación superior, publicista y comunicador social. Se desempeñó como profesor en universidades e instituciones educativas como Cibertec, la Universidad Privada del Norte y la Universidad Señor de Sipán. Colaboró como periodista independiente en las revistas alemanas Digital Development Debates y Tea After Twelve. Integró el equipo organizador de la Feria de Libro de Trujillo como jefe de comunicaciones. Como artista ha realizado exposiciones y eventos culturales. Se desempeña como investigador afiliado a Concytec, habiendo realizado diversas publicaciones académicas.

(2) Laura Bernabé Soto es Licenciada en Artes \& Diseño Gráfico Empresarial y Fotógrafa, con especialidad en Historia del Arte Peruano. Ha expuesto su trabajo y experiencia como fotógrafa en universidades y su investigación "Estudio de la iconografía de la Cultura Lambayeque: Creación de la Marca Región” en el Encuentro Científico Internacional del Norte. Como investigadora egresada de la Universidad Señor de Sipán ha publicado "Diseño de prendas alternativas: proceso y comunicación"..

(3) Julia Beatriz Pelaez Cavero es Educadora en Artes Plásticas y Visuales. Doctora en Comunicación Social, gestora e investigadora especialista en estudios de tipo cualitativos con temáticas sociales, artísticos culturales y ambientales. Es Profesora y Directora de la Escuela de Artes \& Diseño Gráfico Empresarial de la Facultad de Derecho y Humanidades, 
Universidad Señor de Sipán. Es consultora de proyectos culturales y sociales. Participó de exposiciones artísticas y ha sido ponente en diversos congresos, encuentros y seminarios. En su actividad artística y docente ha recibido varios reconocimientos.

\section{Problemática del algodón nativo}

El algodón nativo peruano de la especie Gossypium barbadense L. es una especie hallada de manera espontánea desde épocas precolombinas en Ecuador, Colombia y Perú, así mismo, la especie Gossypium hirsutum L. tuvo presencia en Centroamérica y las variantes Gossypium herbaceum L. y Gossypium arboreum L. en Sudáfrica (MINAM, 2014). La presencia milenaria de esta fibra goza de un arraigo cultural muy marcado entre las comunidades productoras de algodón nativo ubicadas en la costa norte peruana, principalmente en los distritos de Olmos, Jayanca, Illimo, Túcume y Mórrope del departamento de Lambayeque. Estas productoras ven en el de ANC, una actividad que las vincula a sus antepasados Mochica y Lambayeque.

La recuperación del algodón ya no es un tema de preocupación, prueba de ello son las numerosas colectas del pasado reciente promovidas por el Ministerio del Ambiente MINAM que dan fe de ello, pues al revisar la información de los herbarios de la Universidad Nacional de Piura, Universidad Nacional Pedro Ruiz Gallo, Universidad Nacional Mayor de San Marcos y las colecciones del investigador Ola T. Westengen, hallamos 390 colectas realizadas del género Gossypium de las cuales 239 pertenecen a 51 distritos, que forman parte de 19 provincias de 7 departamentos de la costa norte del Perú, como son Tumbes, Piura, Lambayeque, La Libertad, Cajamarca, Ancash y Lima (MINAM, 2014). Además, el MINAM, (2014) en su informe final denominado, Colecta, elaboración de mapas de distribución y estudio socioeconómico de la diversidad del algodón nativo, realizó 109 colectas en 09 regiones del Perú como Amazonas, Cerro de Pasco, Huánuco, Ica, Junín, Lima, Loreto, San Martín y Ucayali, a fin de salvaguardar los recursos genéticos nativos y conocer la real distribución y concentración de los cultivos de algodón nativo y su pariente silvestre el algodoncillo (Gossypium raimondii Ulb.), esta última hallada de manera endémica en el 2019 en el sector de La Colmena entre Chongoyape y la zona de Tocmoche, de acuerdo a lo expresado por el titular del laboratorio general del Instituto de Biotecnología de la Universidad Nacional Pedro Ruiz Gallo, Guillermo Delgado Paredes (Andina, 2019). Así mismo, no es un tema de preocupación el apoyo a las comunidades productoras de AN, pues desde hace doce años los Centros de Innovación Tecnológica de Artesanía y Turismo, CITEs, son entidades que, bajo el ámbito del Ministerio de Comercio Exterior y Turismo, buscan elevar la competitividad del producto artesanal y de los servicios turísticos con el fin de acceder a nichos de mercado nacional e internacional con productos de calidad, diseño novedoso y a precios justos. Por medio de las CITEs, las comunidades productoras son capacitadas en áreas tan diversas que van, desde la mejora de cultivos y aumento de la productividad hasta la capacitación en diseño, comercialización y preparación de catálogos de productos. 
La innovación tecnológica va dejando de ser un problema para el procesamiento e industrialización del algodón nativo. Declercq (2017) propuso en su estudio, Industrialización del algodón nativo peruano de color, el uso de una máquina especialmente adaptada para el proceso de hilado y que responda a las particularidades que el algodón nativo posee como, ser de fibra corta y tener baja resistencia a la torsión, lo que dificulta su hilado con maquinaria convencional. Es conocido también que, uno de los métodos actualmente utilizados para trabajar la fibra nativa, es la combinación con el algodón comercial como el Pima y Tangüis para de esta manera utilizar la máquina de hilado tradicional.

Actualmente y de acuerdo a las evidencias a favor de la puesta en valor del algodón nativo, respaldada por instituciones del gobierno central, regional, e investigaciones realizadas por diferentes universidades, el algodón nativo sigue sin proveer de un beneficio económico sustancial a las asociaciones de comunidades productoras, es decir a aquellas comunidades indígenas, como el caso de la productora Nelly Sánchez Santa María, del caserío Arbolsol en Mórrope, quien manifestó ganar doscientos soles al mes a causa de la pandemia, la mitad de lo que solía obtener en otro tiempo.

Álvaro Becerra, Profesional en la articulación turismo - artesanía de Cite Sipán, menciona que no hay asociaciones productoras de ANC que trabajen sin la Cite, ya que por medio de ellos las asociaciones reciben todo el apoyo para lograr sus metas, sin embargo, da cuenta que pese a el respaldo brindado y la participación en los talleres gratuitos que se les ofrece, reconoce que en la mayoría de los casos les cuesta cambiar la forma tradicional de hacer las cosas, además, les gusta participar de todo el proceso evitando tercerizar alguna parte de la producción.

En lo referente al impacto ambiental en las parcelas de los productores artesanales, se destaca, la no utilización de insecticidas para el control de plagas, el uso de aguas de riego (no potable), el riego moderado que los arbustos necesitan y por ser fibras de color natural, se descarta el uso de cualquier tipo de colorante químico. Es en suma una fibra 100 \% natural. Esta es una diferencia sustancial si consideramos que en los cultivos a gran escala el uso del pesticida en cantidades controladas es importante para asegurar el crecimiento del copo de algodón. "Se hicieron aplicaciones foliares de imidacloprid para combatir las altas infestaciones de fitófagos como: Bemisia tabaci y Aphis gossypii. Los cuales son plagas muy frecuentes en esta especie" (López y Gil, 2017).

Luego del breve repaso de la situación actual del ANC es pertinente entonces preguntarse, el algodón nativo, cuya herencia cultural es innegable, ¿necesita ser revalorado? y si ha de revalorarse, qué aspectos serán convenientes revalorar. Si el aspecto cultural no es suficiente para su puesta en valor, ¿acaso el económico lo será? Al parecer hay dos caminos culturales y económicos bajo un mismo fin. El algodón nativo para un fin económico y el algodón nativo para un fin cultural.

\section{Comunidades productoras, extensión cultural e identidad}

La región Lambayeque es la mayor productora de algodón nativo peruano (Cite Sipán, 2010) Se han encontrado muestras de su domesticación en Huaca Prieta en el Valle de 
Chicama, en la costa norte (Vreeland, 1981) y en Ancón, en la costa central, hace aprox. 2500 años A.C (Brack Egg, 2015). Aunque muchos de los telares hallados no están en muy buen estado, se han encontrado instrumentos textiles como husos, agujas de coser y varas de los telares de cintura que dan cuenta de la labor textil en las antiguas culturas de la costa del Perú (Vreeland, 1985).

En 1551, el cronista Cieza de León señaló que en los valles de Motupe y Pacasmayo existían grandes algodonales. La producción artesanal indígena de tejidos y algodón fue de vital importancia en las actividades económicas en el norte durante la época colonial. Las fuentes escritas de entonces indican que el tejido jugaba un rol socio-económico y cultural muy importante en todo el país (Vreeland, 1985).

Hans Heinrich Brüning, etnógrafo alemán, documentó el algodón durante su estadía en varios distritos de Lambayeque a inicios del siglo XX; él estuvo presente durante la crisis del algodón, cuando no era bien vista su cosecha. En cuanto a la técnica de hilar, realizó algunas anotaciones de nombres de sus herramientas y croquis sobre cómo se realizaba esta labor. Brüning, en su diccionario Muchik revela frases referentes a la técnica de hilar, que se hacía en tres categorías según su grosor. Gracias a sus fotografías y anotaciones, se sabe que la práctica del hilado y tejido era realizado en su mayoría por mujeres indígenas sobre todo en Olmos, Motupe, Jayanca, Túcume y Mórrope, pero que también hubo un considerable número en pueblos mestizos como Chiclayo, Lambayeque y Sechura; principalmente se confeccionaban alforjas, y en menor cantidad colchas, mantas, manteles y fajas (Schaedel, 1988).

En 1949, mediante decreto supremo se empieza a prohibir la cosecha de algodón nativo aduciendo que atraían plagas, reiterándose su prohibición en 1984 y 1994 (Fustamante, 2012). A pesar de las prohibiciones, el algodón nativo logró sobrevivir gracias a que muchas familias indígenas siguieron cultivándolo en sus huertos, usándolo para fines artesanales y medicinales, manteniéndolo vigente y transmitiendo el conocimiento de las técnicas de hilado y tejido de generación en generación.

Tras varias investigaciones y su puesta en valor, el 6 de mayo del 2008 se dispuso la Ley 29224 que declara "Patrimonio genético, Étnico-cultural de la Nación al algodonero nativo peruano", disponiéndose asî "su rescate, recuperación, conservación y promoción en el ámbito nacional" (Fustamante, 2012).

Las comunidades productoras de la región Lambayeque se concentran especialmente en Mórrope, San José, Túcume, Ferrañafe. Cite Sipán tiene empadronadas a 14 asociaciones y talleres que acogen a un total de 182 mujeres artesanas. Algunos grupos están conformados por familiares y otros entre vecinas de la zona, quienes venden sus productos en museos locales o en pequeñas tiendas acondicionadas en sus casas, participan en ferias tanto a nivel local, nacional e internacional. Debido a la coyuntura provocada por el covid-19, las artesanas se han afectado al no poder ofrecer sus productos presencialmente, pese a ello algunas de estas asociaciones y talleres ofrecen y venden sus productos a través de las redes sociales, incluso Cite Sipán las apoya promocionando sus productos en su página de Facebook.

La mujer artesana del algodón nativo no accede a la educación superior, incluso pocas logran a terminar los estudios escolares. Realizan labores domésticas y trabajo en el campo para proveer de mayor ingreso económico a la familia, ya que la actividad artesanal es in- 
suficiente. Las artesanas en su mayoría desean continuar con su labor artesanal, ya que se sienten identificadas con ello, lo valoran y desean seguir transmitiendo los conocimientos que han heredado de sus antepasados (Fustamante, 2012).

Si bien es cierto la tradición y la cultura son claves para ser testigos de las propiedades y uso del algodón, este respaldo es sobre explotado, es decir, se deja de lado otras particularidades alrededor del ANC y se continúa con el discurso cultural, histórico y turístico para valorizarlo. El modo de operar es que, si hay una actividad cultural ésta debe ser promovida al turista nacional y sobre todo al extranjero que es el público objetivo para la venta de los productos, algo viable, pero insuficiente, aún no se vende y promociona el producto artesanal bajo otros conceptos. No por algo los puntos de venta se encuentran en museos y ferias culturales. Son pocas las tiendas en los distritos de Lambayeque que venden estos productos. Se necesita más exposición, comunicación de otros valores y mayor apoyo para establecer un modelo de negocio adecuado a la artesanía.

No obstante, así como exponemos que es insuficiente el beneficio de explotar el valor cultural, debemos reconocer la importancia de la identidad cultural de las artesanas, mujeres muy identificadas con su legado indígena, orgullosas de ello y temerosas de que se pierdan sus costumbres y tradiciones, para las comunidades, el tejido artesanal es tan importante hoy como en el pasado, es a ellas a quienes le debemos la permanencia del algodón nativo. Vreeland (1985) se preguntó cómo algunos aspectos de la cultura autóctona lambayecana han persistido desde épocas prehispánicas y sobre sus cambios. Sin duda, la persistencia en la tradición y las costumbres, ha permitido tener al día de hoy un panorama de lo que es y fue la producción textil en algodón nativo. Y tal como señaló el antropólogo, las investigaciones en algodón nativo deben ayudar a unir el pasado, el presente y el futuro. La cultura que representa el tejido en algodón nativo debe mantenerse, pero si no se llega a materializar un adecuado proceso productivo para hacerla más viable y moderna, quedará relegada y terminará con la última generación de artesanas.

\section{Algodón nativo: características genéticas, competitividad, producción y beneficio medio ambiental}

Tras la promulgación la ley № 29224 la atención hacia el algodón nativo ha sido exponencial, los estudios alrededor de él, nos han permitido conocer sus características genéticas y saber que su fibra es corta y que tiene poca resistencia a la torsión, que sus colores son naturales, que su carga cromosómica es diploide ( $2 \mathrm{n}=2 \mathrm{x}=26$ cromosomas) y en ciertos casos tetraploide $(2 \mathrm{n}=4 \mathrm{x}=52$ cromosomas), que de 7 colores (Verde, pardo, fifo, crema, blanco, marrón, beige) rescatados inicialmente, el investigador Guillermo Delgado Paredes del Instituto de Biotecnología de la Universidad Nacional Pedro Ruiz Gallo (UNPRG) en noviembre de 2018 amplió a 15 el número de colores identificados, dicho material genético se conserva en el banco de germoplasma de la UNPRG (Andina, 2018).

También sabemos que las especies predominantes en Lambayeque son el Gossipyum barbadense L. con un 71\% de presencia y Gossipyum hirsutum L. con un 5.4\% (MINAM, 2014). Además, en consecuencia, de la puesta en valor del algodón nativo, varios estudios 
toman como eje las posibilidades económicas que traería el uso de la fibra nativa, como la investigación Línea artesanal de tejidos en algodón nativo patrocinada por el MINCETUR y Cite Sipán (2010) cuyo objetivo fue analizar el estado actual de la línea artesanal de tejidos en algodón nativo y su potencial de desarrollo económico en Lambayeque. Mencionaremos además el estudio, Reintroducción de algodón nativo (Gossypium barbadense) en la costa norte del Perú: Análisis de factibilidad económica para pequeños productores (2015) En el cual los estudios de factibilidad asegurarían que 50 agricultores que destinasen el 10\%, $25 \%$ e incluso el $50 \%$ de su parcela al cultivo del ANC los resultados serían favorables a su ingreso económico.

Bajo la misma línea Estrada, et al., (2016) Estudio sobre las oportunidades para comercializar prendas de vestir de algodón nativo, nos permite entender que es posible ampliar el ámbito de producción y difusión cultural no solo al algodonero nativo y las asociaciones productoras, sino que es factible que personas independientes interesadas en esta fibra, formen parte del potencial económico que esta ofrece. De este modo se lograría la tan ansiada industrialización, volcando a la práctica todo el conocimiento acumulado en una década de investigaciones a favor de su reconocimiento a nivel nacional, puesto que se ha comprobado que el ANC no es un hospedador de plagas incontrolables como se difundió en el pasado, por el contrario, resistente a variedad de plagas, males bacterianos y fungosos, y en condiciones naturales, soporta suelos salitrosos, su cultivo no requiere de gran consumo de agua, tanto que en condiciones extremas y suelos arenosos llega a soportar hasta cinco años sin riego (Vásquez y Pérez, 2011). No olvidemos también que el color de su fibra no proviene de ningún colorante artificial, por estas características físicas se convierte en una planta que con voluntad y organización podría extenderse con rapidez en cualquier suelo geográfico del país.

Se estima que un arbusto de algodón nativo da en promedio $1 \mathrm{~kg}$ de fibra natural y si bien es cierto, aun no se sabe con seguridad a cuántas hectáreas asciende la producción de ANC en Lambayeque, Declercq, (2016) en su investigación, Estudio de pre-factibilidad para la instalación de una planta productora de hilos de calidad elaborados con algodón nativo de color, nos da a conocer por medio de la entrevista realizada al Ing. Percy Vásquez presidente de Asociación distrital de productores de maíz amarillo duro y otros cultivo de Mórrope - Aspromad, que el algodón nativo de color se cosecha 2 veces al año en los meses de mayo y junio con un rendimiento promedio de $1000 \mathrm{~kg} / \mathrm{hectárea;}$ y en los meses de octubre y noviembre un rendimiento promedio de $2000 \mathrm{~kg} /$ hectárea; por lo tanto la producción anual es de $3000 \mathrm{~kg} /$ hectárea. Un dato que contrasta con la experiencia de la señora Nelly Sánchez, quien cosecha fibra tres veces al año en su parcela. Por otro lado, según indican Ocampo y Vásquez el incremento de la producción anual de ANC aumentó significativamente, de 15 TM en el 2010 a 150 TM en el 2013 (Ocampo y Vásquez, 2015 citado en Declerq, 2016) Como vemos hay una diferencia abismal entre personas que tienen la visión comercial del ANC y las que mantienen el arraigo cultural pausado de la producción. La Sra. Nelly Sánchez principalmente cultiva maíz, uva, cría animales menores como gallinas y cerdos, y también posee algunas vacas. Al ser consultada sobre que otras actividades desarrolla cuando no tiene colectas de fibra natural, nos dice que recurre a los hilos industriales para hacer otro tipo de productos. Algo que llama la atención, siendo ella una 
persona que se dedica a la elaboración de productos como bolsos, monederos, tapetes, llaveros de fibra natural por más de veinte años.

Esto ocasiona que la valoración del algodón nativo quede en manos del visitante, del turista, quien se admira por la historia detrás de la fibra y de quienes se dice son los que mejor aprecian los productos en comparación con los locales. Sería pertinente aumentar la producción de algodón en las parcelas de las productoras, para volverse tan competitivas como los que la compran y comercializan en Lima ganando mucho más en el mercado nacional e internacional.

\section{Diseño regional de productos e innovación en diseño para exportación}

Uno de los grandes retos de la artesanía es adaptarse al mundo contemporáneo sin perder su esencia ni desaparecer; tiene múltiples dificultades, tanto en el posicionamiento como en la competencia, en la gestión de procesos de producción y distribución y en la comercialización (Cruz et al, 2019). El diseño en la artesanía tiene tres aspectos importantes: "el cultural como el rescate de los valores, el técnico-formal con el desarrollo de formas, funciones, materiales y técnica, y el socioeconómico en el progreso justo del sector productivo" (p. 133) (Corral, 2002). También, ambos tendrían tres sentidos de interrelación: el de tradición, que busca la preservación de técnicas y objetos; el de desarrollo, que consiste en la aplicación de técnicas de mejora y producción para hacer crecer la actividad artesanal y la economía de las comunidades; y el de innovación, que busca la generación de nuevas ideas y renovación de productos (Ariza y Andrade, 2021).

El diseño en la artesanía se posiciona como una disciplina capaz de resolver problemas de fondo, convirtiéndose en un instrumento de emancipación (Martínez-Osorio et al, 2020). De esta forma el diseño elevaría de manera múltiple el potencial artesanal, además de corregir ciertos problemas en cuanto a su producción, y, sobre todo, la continuidad de la cultura y tradición que posee.

Los productos artesanales en algodón nativo son diseñados enteramente por las artesanas. Ellas continúan elaborando tal y como hace cientos de años alforjas, fajas, manteles y, con el tiempo se han adaptado, haciendo nuevos productos como bolsos, morrales, llaveros, monederos, bufandas, pulseras, porta celulares, entre otros. La técnica ancestral es el telar de cintura, pero también realizan tejidos a crochet, a palillo; costuras y bordados a mano. El diseño de sus tejidos posee motivos geométricos como rombos y cuadros, pero sobre todo líneas y en menor medida iconografías de la cultura Moche y Lambayeque, como olas, aves en picada, figuras escalonadas y otras formas geométricas, a veces realizan diseños complejos y más elaborados. En general, el desarrollo en diseño es bajo provocando un menor flujo de ventas, Fustamante (2012) señala:

Mejorando el acabado de los artículos de artesanía y adecuando los diseños a los gustos y preferencias de los consumidores se logrará el ingreso a mercados más exigentes y con mayores márgenes de ganancias. Los accesorios de vestir, artículos de decoración y regalo, así como, la bisutería que son rubros donde 
las artesanías en algodón nativo encajan, cuentan con importantes mercados en Francia, Alemania y Estados Unidos (p. 82).

El producto artesanal es diseñado y hecho a mano, es cuidadoso y perfeccionado, se atienden los detalles, incluso tienen variaciones propias por el trabajo manual; además las técnicas heredadas de antepasados hacen que los productos artesanales tengan un valor diferencial y único por cada pieza, más aún si es seriada, constituyendo en definitiva un valor de marca (Velasco, 2015), valor que debe mantenerse vigente con la constante innovación en diseño. Pero las artesanas continúan la misma línea de diseño, realizando productos repetitivos.

Álvaro Becerra, Profesional en la articulación turismo - artesanía - Cite Sipán de Lambayeque, menciona la falta de calidad en los productos de algodón nativo, debido a que los artesanos son muy tradicionales y están presentes en todo el desarrollo de la producción de manera individual y sin asistencia, pese a no ser expertos en cada fase del proceso de fabricación. Indica que existe poco interés en el diseño por parte de las artesanas, por ejemplo, se les ha dado algunas recomendaciones para modernizar los diseños de los tejidos, como la utilización de patrones, ya que ha observado en diferentes ferias la preferencia de los compradores por tejidos con diseños elaborados antes que tejidos llanos, pero no los realizan pues les demanda más tiempo. Becerra considera importante una capacitación en diseño textil, como complemento a la escasa paleta de colores que tiene el algodón nativo; de la misma forma sucede con la presentación del producto, las artesanas continúan con la idea de seguir apartándose del diseño de etiquetas y empaques a fin de abaratar costos. Cite Sipán ha brindado capacitaciones en cuanto a desarrollo de productos y realizando eventos de moda que han concluido con pasarelas, donde las prendas fueron realizadas por diseñadores de modas, quienes además capacitaron a las artesanas en confección de prendas. El apoyo de esta institución ha sido importante para el avance en la innovación de los productos artesanales en los últimos doce años.

Asimismo, el diseño contribuiría a la posibilidad de exportación de los productos artesanales; hasta hoy ninguna artesana ha tenido la oportunidad de exportar su trabajo, sin embargo, cuando se han llevado sus productos a ferias en el extranjero con el apoyo de instituciones públicas, se ha notado un mayor ingreso por ventas (Fustamante, 2012). En el estudio Propuesta de comercialización para fomentar la exportación de mantas de algodón nativo de colores de la Cite Sipán al mercado de Alemania, 2020, se recalca de la importancia del diseño Moche en el producto para la exportación a dicho país, también del diseño de su etiquetado y empaquetado, además de que existe la tendencia hacia una paleta de color de fibras naturales (Diaz, 2020).

Es necesario capacitar a las artesanas, no solo en diseño, sino también explicarles porque es importante el proceso del diseño en la artesanía y de cómo esto no va a afectar sus tradiciones.

El diseño hoy puede trabajar de la mano con lo tradicional, generando una fusión del pasado con lo contemporáneo, sin perder su esencia, prueba de ello es la diferencia entre los productos de las artesanas de Mórrope y Túcume. El museo de sitio de Túcume lleva años realizando capacitaciones a numerosos artesanos, entre ellos a las de algodón nativo, promoviendo su producción y diseño, también han recibido capacitaciones del Grupo Axis, 
Arte investigación aplicada al desarrollo, de la Pontificia Universidad Católica del Perú en el marco del Proyecto "AXIS TÚCUME: Reconstrucción del vínculo cultural entre el patrimonio prehispánico y la comunidad. Recuperación de la iconografía y su aplicación en productos artesanales", estas capacitaciones han sido la clave, haciéndolos más atractivos en comparación con los productos de las artesanas de Mórrope, quienes acuden a las capacitaciones de manera discontinua y poco participativa, prefiriendo la labor enseñada por sus abuelas y madres.

\section{Comunicación para la revalorización y sustentabilidad}

El aporte final de este ensayo nos ofrece una nueva perspectiva, principalmente sobre la forma en que se difunde el algodón nativo de color que, si bien es cierto, a partir de su puesta en valor y respaldo legislativo del 6 de mayo de 2008 ha contribuido al fortalecimiento del algodonero nativo y su herencia cultural materializada en los copos del algodón G. barbadense L. y su relación con las comunidades productoras, ha llegado el momento, a nuestro parecer, de evaluar todo lo realizado en los puntos que a continuación detallamos, con el propósito de avanzar a la consolidación económica, sociocultural y ambiental del algodón nativo, no solo en la costa norte peruana sino que se pueda extender con nuevos brillos a nivel nacional en los próximos años.

\section{Virtudes físicas y genéticas del ANC}

Difundir de manera sostenida que por su característica genética el algodón nativo posee una coloración natural que a la fecha consta de 7 colores y que podrían ascender a 15, si se trabaja adecuadamente con el material genético que resguarda la UNPRG.

Difundir de manera sostenida que la especie Gossypium crece de manera segura en suelos áridos y es capaz de soportar condiciones climáticas diversas, prueba de ello es que se encuentra en costa sierra y selva y que sus primeros hallazgos fueron silvestres y espontáneos, es decir crecieron de manera natural en los campos.

Difundir permanentemente en las comunidades productoras información relevante y actualizada de las características físicas de la fibra de algodón nativo, pues un productor informado, potenciaría su capacidad competitiva a mediano plazo.

\section{Beneficio ambiental}

Informar adecuadamente en el campo agrícola y comercial que el algodón nativo de color producido por las asociaciones productoras, son producciones a pequeña escala y que por ello su característica es natural, con bajo consumo de agua (no potable), libre de pesticidas y colorantes químicos, y en beneficio de personas con alegrías en la piel, para así revertir la mala fama que por décadas se le dio al ANC.

Asimismo, reforzar el conocimiento sobre técnicas de conservación del suelo, el control del agua y el constante control de plagas utilizando productos naturales inofensivos a 
los trabajadores. El cultivo del algodón agroecológico establece una relación en la que se mitiga el impacto a la naturaleza y donde los principales beneficiarios son los que actúan directa o indirectamente en su producción (FAO; ABC/MRE, 2018).

\section{Innovación en diseño}

Comunicar estratégicamente a las asociaciones productoras de ANC, que la incorporación de nuevos diseños acordes con las preferencias y gustos de los visitantes les sumará beneficios económicos y una nueva manera de comunicarse con los nuevos mercados sin perder su valiosa identidad cultural.

\section{Desarrollo económico y sustentabilidad}

Invitar a las asociaciones productoras de ANC a confiar en la herencia cultural y unidad familiar de la que gozan para dar el siguiente paso, aplicando todo lo aprendido y manteniendo comunicación constante con sus líderes y el compromiso a favor del porvenir familiar. Ayudando al desarrollo sustentable de las organizaciones involucradas con una producción comunal y producción industrial, haciendo posible una rentabilidad económica social y ambiental tanto para los productores artesanales como para los inversores.

\section{Referencias bibliográficas}

Andina (2019, 9 de julio). Descubren plantas de algodón de fibra verde endémica en Lambayeque. Consultado el 11 de febrero de 202. https://andina.pe/agencia/

Andina (2018, 28 de noviembre). Investigadores identifican 15 colores naturales de algodón nativo de Lambayeque. Consultado el 13 de febrero de 202. https://andina.pe/agencia/

Ariza, V., y Andrade, M. (2021). La relación artesanía y diseño. Estudios desde el norte de México. Cuadernos del Centro de Estudios en Diseño y Comunicación. Ensayos, (90), 193-211.

Brack Egg, A. (2015). Perú. Catorce recursos genéticos que cambiaron el mundo y uno que lo cambiará. Promperú.

Cite Sipán (2010). Línea artesanal de tejidos en algodón nativo. Lima: MINCETUR.

Corral, R. (2002). Gestión de diseño y planeación estratégica en el contexto artesanal. Revista Artesanías de América, (52), pp. 125-144.

Cruz, C.; González, A. y Fernández, F. (2019). Hacia un ontológico de Creatividad aplicado al contexto del Diseño y Artesanía: Innovación y Computación Evolutiva. i+ Diseño. Revista científico-académica internacional de innovación, investigación y desarrollo en Diseño, (14), 266-276. https://doi.org/10.24310/Idiseno.2019.v14i0.7109

Declercq, L. (2016). Estudio de pre-factibilidad para la instalación de una planta productora de hilos de calidad elaborados con algodón nativo de color. Trabajo de investigación para optar el título profesional de Ingeniero Industrial. Universidad de Lima.

Declercq, L. (2017). Industrialización del algodón nativo peruano de color. Ingeniería Industrial $N^{\circ} 35$, enero-diciembre 2017, ISSN 1025-9929, pp. 141-161. 
Diaz, F. (2020). Propuesta de comercialización para fomentar la exportación de mantas de algodón nativo de colores de la CITE Sipán al mercado de Alemania, 2020. Global Business Administration Journal 4(1), 2020.

Estrada, A; Tinoco, L.; Chamay, K.; Quiñonez, S.; Alfaro, R.; Moya, S. y Arca, E. (2017). Estudio sobre las oportunidades para comercializar prendas de vestir de algodón nativo. Facultad de Ciencias Económicas y Empresariales. Lima: Global Business Administration Journal 1(1), 2017.

Fustamante, K. (2012). Procesamiento artesanal del algodón nativo: una actividad económica viable o sólo una tradición. Tesis de Maestría Pontificia Universidad Católica del Perú. Repositorio Digital de Tesis y Trabajos de Investigación PUCP.

López, E. y Gil, A. (2017). Fenología de Gossypium raimondii Ulbrich "algodón nativo" de fibra de color verde. Scientia Agropecuaria 8 (3): 267-271 (2017)

Martínez-Osorio, P. A.; Paschoarelli, L. C., \& Da-Cruz-Landim, P. (2020). Diseño y artesanado: una mirada contemporánea. Revista de Arquitectura (Bogotá), 22(1), 130-137. https://doi.org/10.14718/RevArq.2020.1975

Ministerio del Ambiente. (2014). Distribución y concentración de las razas locales de algodón nativo en la Costa Norte del Perú. Lima: Ministerio del Ambiente.

FAO; ABC/MRE. (2018). Estudio nichos de mercados del algodón cooperación sur-sur trilateral. Santiago de Chile.

Schaedel, R. P. (1988). La etnografía Muchik en las fotografías de H. Brüning 1886-1925. Ediciones COFIDE.

Vásquez, P., y Pérez, P. (2011). Revalorando un cultivo ancestral. Algodón nativo: fibra de calidad para la industria. Chiclayo, Perú: Cáritas.

Velasco, R. (2016). El valor sensible de la artesanía en el diseño. i+Diseño. Revista científico-académica Internacional De innovación, investigación Y Desarrollo En Diseño, (14) 91-98. https://doi.org/10.24310/Idiseno.2016.v11i0.2410

Vreeland, J. (1981). Algodón de colores: la novedosa planta Antigua. CIID informa, v. 10, no. 2. Vreeland, J. (1985). Algodón y arte textil en Lambayeque, ayer y hoy. En: Mendoza Santillán, E. (Ed.), Presencia histórica de Lambayeque (pp. 142-154). Chiclayo: Ediciones y Representaciones H. Falconí.

\begin{abstract}
The essay approaches the issue of Peruvian colored native cotton and its use in the elaboration of artisan products that cannot compete with commercial cotton. Nevertheless, families of the producing communities see in cotton a cultural extension to keep in force, being this insufficient for its valuation. The aim is to propose the cotton revaluation, from the communication of its genetic virtues, the environmental benefit of its production and design innovation towards the contemporary market, guaranteeing that its pre-Columbian essence enhances the economic development of the producing communities within the framework of sustainability and identity.
\end{abstract}


Keywords: native cotton - producing communities - crafts - design - sustainability - revaluation.

Resumo: O texto trata sobre o problema do algodão colorido peruano nativo e seu uso na elaboração de produtos artesanais, o qual não pode competir com o algodão industrial. Apesar disso, as famílias das comunidades produtoras veem no algodão uma extensão cultural a manter em vigor, ainda que não seja suficiente para sua valorização. O objetivo é uma proposta de revalorização do algodão, a partir da comunicação das suas virtudes genéticas, do benefício ambiental na sua produção, e da inovação em design para o mercado comteporâneo, garantindo que sua essência pré-colombiana potencialize o desenvolvimento econômico das comunidades produtoras do quadro de sustentabilidade e identidade.

Palavras chave: algodão nativo - comunidades produtoras - artesanato - design sustentabilidade - reavaliação.

[Las traducciones de los abstracts fueron supervisadas por el autor de cada artículo] 